\section{Influenza - was bringen die Neuraminidase-Hemmer?}

\author{
B. Salzberger ${ }^{1}$ \\ ${ }^{1}$ Klinik I für Innere Medizin, Universitätsklinikum Regensburg
}

Die Influenza (A und B) tritt seit vielen hundert Jahren fast jährlich epidemisch und in unregelmäßigen Abständen auch pandemisch (fast ausschließlich Influenza A) auf. Die Influenza kann bei nichtimmunen Personen, insbesonders bei Immundefekten oder -defizienzen, schwer verlaufen, meistens als Infektion der tiefen Atemwege.

Es sind seit vielen Jahren sichere Vakzinen entwickelt worden, die aufgrund der sehr typspezifischen und kurzen Immunität und der sich ständig ändernden Virustypen jährlich wiederholt werden müssen. Insbesonders bei immundefizienten und älteren Personen ist die Wirksamkeit der Impfung von nur $30-50 \%$ unbefriedigend. Für diese Patienten und auch generell für schwere Verläufe ist eine antivirale Therapie eine wichtige Behandlungsoption. Die einfachste Definition einer schwer verlaufenden Influenza ist die einer stationär behandlungspflichtigen.

Eine Reihe von antiviralen Substanzen ist bisher zur Therapie evaluiert worden, initial Amantadin und Rimantadin, später die gezielt entwickelten Neuraminidase-Inhibitoren (zunächst Oseltamivir und Zanamivir). Alle diese Substanzen sind in-vitro gut antiviral wirksam, Amantadin und Rimantadin sind allerdings gegen die in den letzten Jahren zirkulierenden Stämme wirkungslos. Die klinische Prüfung der Neuraminidase-Inhibitoren ist bei Patientenkollektiven mit einem geringen Komplikationsrisiko und nur wenigen schwer verlaufenden Influenzafällen durchgeführt worden. Zusammengefasst haben diese Studien gezeigt, dass Neuraminidase-Inhibitoren bei rechtzeitigem Einsatz (innerhalb von $48 \mathrm{~h}$ nach Symptombeginn) die Dauer der Symptome verkürzen und auch als Postexpositionsprophylaxe wirksam sind.

Ein offensichtlicher Kritikpunkt an diesen Ergebnissen war immer die fragliche klinische Relevanz dieser Ergebnisse: Rechtfertigt die Verkürzung einer unkomplizierten Erkrankung den Einsatz einer antiviralen Substanz?

Ob eine relevante klinische Wirksamkeit der NeuraminidaseInhibitoren auch bei einem Risikokollektiv von Patienten gegeben ist, wurde nie in einer spezifischen Studie untersucht, allerdings wurde der Einsatz von Neuraminidase-Inhibitoren bei Risikopatienten und schweren Verläufen in Hinsicht auf die in-vitro- und klinische Wirksamkeit bei Nicht-Risikopatienten breit durchgeführt und empfohlen.

Eine Reduktion der Häufigkeit von Komplikationen wurde in Metaanalysen der Zulassungsstudien nachgewiesen, allerdings mit unterschiedlichen Ergebnissen je nach Einschluss verschiedener Studien. Bei sorgfältigen Analysen fanden sich zudem Differenzen zwischen den veröffentlichten Studien- und den Rohdaten dieser Studien. Diese Diskrepanzen führten zu einer großen Skepsis bezüglich der Qualität der Studien und damit auch zur Diskussion über die Wirksamkeit von Neuraminidase-Inhibitoren. Eine internationale Kollaboration von Epidemiologen hat die beteiligten Firmen in zähen Verhandlungen letztlich erfolgreich dazu gedrängt, die vollständigen Datensätze dieser Studien zur Verfügung zu stellen. In der Metaanalyse der als hochwertig einzustufenden Studien fand sich die Bestätigung der Reduktion der Symptomdauer, allerdings kein Einfluss auf das Auftreten schweren Komplikationen. Diese waren allerdings im gesamten Datensatz der Metaanalyse sehr selten und zudem auch nicht gut prospektiv definiert worden - eine Situation, in der ein solcher Nachweis auch extrem schwierig ist. Diese Ergebnisse wurden weltweit in der Laienpresse breit diskutiert. Sie wurden verständlicherweise als Kritik an der damaligen Zulassungspraxis genutzt, in der wichtigsten offenen Frage allerdings mit einem Kurzschluss in der Interpretation: der Nichtnachweis der Wirksamkeit wurde als Nichtwirksamkeit ausgelegt und publiziert.

Die Pandemie im Jahre 2009 bot die Möglichkeit einer retrospektiven Analyse der Wirksamkeit von Neuraminidase-Inhibitoren in Bezug auf schwere Verläufe. Hiermit zeichnet sich ein anderes Bild ab: In einer Metaanalyse von Studien mit schweren Verläufen zeigte sich ein Nutzen durch die Behandlung. Ein signifikanter Effekt auf das Überleben war vor allem bei frühem Behandlungsbeginn erkennbar, ein Nutzen war aber auch noch bei einem Intervall von 5 Tagen zwischen ersten Symptomen und Therapiebeginn vorhanden - also deutlich länger als bisher gedacht. Diese Evidenz für die klinische Wirksamkeit von Neuraminidase-Inhibitoren hat nicht die Qualität einer guten randomisierten Studie, wird aber für die nächsten Jahre vermutlich die beste Evidenz bleiben und damit auch den Therapiestandard definieren.

Neben diesen offenen klinischen Fragen zur Wirksamkeit sind andere Fragen ebenfalls offen: Wie ist die Dynamik der Virusreplikation in den einzelnen Zellkompartimenten und wie wird sie durch antivirale Substanzen verändert?

Diese Fragen sind z.B. für die HIV-Infektion gut charakterisiert - allerdings sind hier auch die Kompartimente, in denen die Replikation stattfindet oder gemessen werden kann, besser charakterisierbar und untersuchbar (Immunzellen bzw. peripheres Blut). Für die Influenza gibt es hierzu nur mathematische Modelle, deren experimentelle Grundlagen unsicherer sind. Allerdings zeigen diese Modelle auch, dass vermutlich nur Kombinationstherapien von verschiedenen Substanzen in der Lage sein dürften, rasch eine fast vollständige Hemmung der Replikation zu erzielen und damit auch eine Entwicklung von sekundären Resistenzen zu verhindern. Klinische Studien zu solchen Kombinationstherapien sind mittlerweile in der Phase II und dürften vor allem für die Entwicklung neuer und zusätzlich wirksamer Anti-Influenza-Substanzen eine wichtige Rolle spielen.

Interessenkonflikte: Teilnahme an Studien GSK zur Wirksamkeit von Zanamivir (Sponsor GSK), Beratertätigkeit für GSK 


\section{Literatur}

1 Jefferson T, Jones $M$, Doshi Pet al. Oseltamivir for influenza in adults and children: systematic review of clinical study reports and summary of regulatory comments. BMJ 2014; 348: g2545

2 Heneghan CJ, Onakpoya I, Thompson M et al. Zanamivir for influenza in adults and children: systematic review of clinical study reports and summary of regulatory comments. BMJ 2014; 348: g2547

3 Muthuri SG, Venkatesan S, Myles PR et al. Effectiveness of neuraminidase inhibitors in reducing mortality in patients admitted to hospital with influenza A H1N1pdm09 virus infection: a meta-analysis of individual participant data. The Lancet Respiratory Medicine 2014;

4 Perelson AS, Rong L, Hayden FG. Combination antiviral therapy for influenza: predictions from modeling of human infections. J Infect Dis 2012; 205: $1642-1645$
Bibliografie

DOI http://dx.doi.org/10.1055/s-0033-1358036

Drug Res 2014; 64, Suppl. 1: S20-S21

(c) Georg Thieme Verlag KG Stuttgart · New York . ISSN 2194-9379

Korrespondenzadresse

Prof. Dr. Bernd Salzberger

Universitätsklinikum Regensburg

93042 Regensburg

bernd.salzberger@klinik.uni-regensburg.de 